\title{
LA INTERNACIONALIZACIÓN DE LOS SERVICIOS TURÍSTICOS EN ANDALUCÍA: CARACTERÍSTICAS Y FACTORES DETERMINANTES
}

\author{
MARTA MUÑOZ-GUARASA \\ Departamento de Economía. UNIVERSIDAD DE JAÉN, ESPAÑA \\ e-mail:mmunoz@ujaen.es \\ ENCARNACIÓN MORAL PAJARES \\ Departamento de Economía. UNIVERSIDAD DE JAÉN, ESPAÑA \\ e-mail: emoral@ujaen.es
}

\section{RESUMEN}

El objetivo de este trabajo es conocer algunos de los factores determinantes y las características del proceso de internacionalización de los servicios turísticos en Andalucía, de acuerdo con paradigma ecléctico OLI (Dunning, 1977, 1993). En la región andaluza no existen datos oficiales de las empresas internacionalizadas. Por ello hemos realizado una encuesta destinada a 130 empresas turísticas internacionalizadas de estas actividades situadas en la región. Los resultados ponen de manifiesto que las ventajas de propiedad consideradas más relevantes por estas empresas son la calidad del servicio, la imagen que tienen los consumidores de ellas, la competitividad en precios y la cualificación del personal. Respecto a las ventajas de localización que creen más importantes son la "proximidad al cliente" y "seguimiento al cliente" y también las características de los mercados: tamaño y crecimiento.

Palabras clave: servicios turísticos, exportaciones, inversión en el exterior, internacionalización.

\section{The internationalization of tourist services in andalusia: features and determinants}

\begin{abstract}
The objective of this study is to learn some of the determinant factors in the internationalization of tourist services in Andalucía in accordance with the Eclectic Paradigm (OLI) (Dunning, 1977, 1993). The study will focus on Andalusia in Southern Spain. In the Andalusian region there are no official data on internationalized companies. We have therefore conducted a survey of 130 internationalized tourist companies within the region. The results show that the ownership or specific advantages considered by the businesses themselves are the quality of service and the image that customers have of the company, price competitiveness and the professional training of employees. Regarding the advantages of locating abroad that are believed to be most important, those companies that engage in foreign investment highlight customer follow-up and finding new customers. As well, they greatly value those factors related to the destination market: size, and growth.
\end{abstract}

Key words: tourist services, export, foreign direct investment, internationalization.

Clasificación JEL: F10, F21, L8 


\section{INTRODUCCIÓN}

El proceso de internacionalización a nivel mundial ha sido liderado por el sector industrial. Sin embargo, en las últimas décadas se ha producido una expansión de este proceso en el sector servicios. Ambos hechos se tratarán de justificar seguidamente. Las características de los servicios, que tradicionalmente se han basado en que estos son intangibles, perecederos, el consumo suele ocurrir de manera simultánea a la producción (Hill, 1977) han condicionado y ralentizado el proceso de internacionalización del sector. Este último rasgo, en el que muchas de las actividades terciarias tienen que producirse y consumirse en el mismo lugar y al mismo tiempo, ha hecho que la vía de entrada en mercados foráneos haya tenido que ser a través de la inversión directa extranjera. Sin embargo, la introducción de las nuevas tecnologías de la información y de la comunicación han permitido separar dicho proceso, de forma que, por ejemplo, actividades como la consultoría, o en general, los servicios empresariales, puedan producirse en un país y consumirlos en otro, y por tanto, posibilitar las exportaciones. Asimismo, la aparición de otras figuras contractuales como las franquicias, contratos de gestión, joint-ventures, etc., han favorecido el proceso de expansión en el exterior de las empresas de servicios. Igualmente, otros factores como la liberalización de muchas actividades del sector servicios a nivel mundial, así como el crecimiento de la demanda de estos productos a nivel nacional han colaborado con el auge del proceso de internacionalización del sector terciario recientemente.

Erramilli (1990) distingue entre los servicios en que la producción y el consumo están por completo separados (hard services), como programas informáticos, discos de música, libros, etc., que se pueden exportar de forma similar a las mercancías y aquellos en los que necesariamente la producción y el consumo ocurren al mismo tiempo (soft services). En este sentido se han realizado numerosos estudios que han tratado de mostrar las características de los diferentes servicios y, de acuerdo con ellas se han tratado de relacionar con las formas de suministro internacional (Sampson y Snape, 1985; Boddewyn et al.,1986; Grubel, 1987; Bhagwati, 1988; Vandermerwe and Chadwick, 1989; Erramilli, 1990; Eduardsson et al., 1993; Sapir, 1993a y 1993b; Roberts, 1998).

En el caso del sector turístico, en el que en este trabajo hemos incluido los servicios de alojamiento, servicios de comida y bebidas y agencias de viajes, algunas de las actividades son hard services como es el caso de las dos primeras y soft services en el caso de la última. Así, las características de la propia actividad pueden ser determinante en la vía de entrada elegida y en los factores que llevan a las empresas a utilizar diferentes estrategias de expansión internacional.

Teniendo en cuenta este contexto, este trabajo tratará de conocer las características del proceso de internacionalización de los servicios turísticos en Andalucía (España) y sus factores determinantes. La estructura del trabajo es la siguiente: Tras esta introducción en la sección segunda se hará un repaso de la literatura económica, que nos ha servido de base para el estudio empírico. La tercera sección se dedicará a la metodología utilizada, que se ha centrado en una encuesta a empresas internacionalizadas, y la cuarta al estudio empírico y los resultados. El trabajo finaliza con unas conclusiones.

Dentro del sector servicios la hostelería alcanzó un 10,5 por 100 de la producción de servicios en España en 2009, porcentaje muy superior al que representa en la UE (4,1 por 100). En el caso de Andalucía la importancia relativa de la producción de estas actividades algo mayor a la nacional, suponiendo un 10,9 por 100 sobre el total del sector servicios en la región. Sin embargo, este gran protagonismo del sector turístico en Andalucía no se aprecia en el proceso de internacionalización del mismo, en el que la inversión directa en el exterior no llega a superar en 2010 un 0,05 del total de la IDE en los servicios.

\section{APORTACIONES TEÓRICAS}

Uno de los objetivos de este trabajo es conocer los factores determinantes del proceso de internacionalización de las empresas turísticas andaluzas. Para ello nos basaremos en la Teoría ecléctica propuesta por Dunning $(1977,1993)$. 
De acuerdo con Dunning (19977) las formas tradicionales de internacionalización de la empresa son: la exportación, la inversión directa y la concesión de licencias de producción a firmas extranjeras. Dunning considera que para que una empresa se internacionalice mediante estas vías debe cumplir una serie de condiciones, las cuáles se presentan en la tabla 1.

1) La empresa debe poseer ventajas de propiedad (Ownership Advantages)- a partir de ahora también ventajas específicas o ventajas competitivas- respecto a sus competidores del país extranjero e incluso respecto a sus competidores domésticos. Estas ventajas se asocian con activos intangibles de la empresa tales como tecnología más avanzada, economías de escala, mejor conocimiento de las técnicas de comercialización y distribución, etc. Para que éstas puedan ejercer una influencia es necesario que permanezcan de forma exclusiva en la firma durante un período de tiempo suficientemente largo.

2) La empresa debe poseer ventajas de internalización (Internalization Advantages) para poder internacionalizarse mediante las exportaciones o la inversión directa extranjera. La condición primera no es suficiente, es necesario que la empresa tenga el deseo y la capacidad de internalizar esa ventaja específica. Ello le resulta beneficioso en la medida en que se reducen los costes de transacción del mercado.

3) Si la empresa se decide por invertir en un país determinado, éste debe ofrecer ventajas de localización (Location Advantages) respecto al país de origen. Estas ventajas no están solamente asociadas a los costes y a la disponibilidad de los factores de producción o al mercado, sino también a las particularidades del sistema institucional instaurado en el país receptor de la inversión (sistema fiscal, reglamento laboral, grado de restricción a los intercambios, integración en áreas supranacionales, etc.).

Tabla 1

Elección de la forma de internacionalización

\begin{tabular}{|c|c|c|c|c|}
\hline & & \multicolumn{3}{|c|}{ Ventajas } \\
\hline \multirow{4}{*}{$\begin{array}{l}\text { Formas de } \\
\text { Internacionalización }\end{array}$} & & $\begin{array}{c}\text { Propiedad de la } \\
\text { empresa }\end{array}$ & Internalización & Localización \\
\hline & $\begin{array}{c}\text { Inversión en el } \\
\text { exterior }\end{array}$ & SI & SI & SI \\
\hline & Exportación & SI & SI & NO \\
\hline & Licencia & SI & $\mathrm{NO}$ & $\mathrm{NO}$ \\
\hline
\end{tabular}

Fuente: Dunning (1988: 28)

Estas ventajas se configuran como el «Paradigma OLI», el cual vincula la decisión de invertir en el extranjero a las características propias de la empresa, a las del sector y a las del país de acogida de la inversión. Cada uno de los conceptos integrados en el «Paradigma OLI» abarca diferentes teorías propuestas acerca de las causas que motivan la inversión directa extranjera:1) Ventajas específicas o de propiedad: Teoría de la organización industrial. 2) Ventajas de internalización: Teoría de los costes de transacción y 3) Ventajas de localización: Teoría de la localización ${ }^{\bullet}$

Estos estudios teóricos y empíricos se han centrado fundamentalmente en la industria, mientras que los del sector terciario han sido bastante escasos. Sin embargo, en estas últimas décadas, con el auge del comercio de servicios, están proliferando los estudios dedicados a dicho sector (Guerrieri y Meliciani, 2007; Jenícek, 2007; Muñoz-Guarasa, 2007; Sánchez-Peinado, Pla-Barber y Hébert, 2007; Kolstad y Villanger; 2008; Ramasamy y Yeung, 2010), aunque todavía siguen siendo limitados, lo que justifica la elaboración de investigaciones tendentes a conseguir un mayor conocimiento del proceso de internacionalización de los servicios $\mathrm{y}$, en especial, al de los servicios turísticos.De acuerdo con Boddewyn et al. (1986) y Buckley et al. (1992), la Teoría ecléctica (Dunning, 1977) puede ser aplicable a las empresas de servicios. Sin embargo, otros cuestionan dicha aplicabilidad

\footnotetext{
- Un repaso de las principales aportaciones teóricas se pueden ver en Muñoz-Guarasa (1999: 47-92)
} 
(Johanson y Valhne, 1990 y O'Farrell et al.,1996) y reclaman la necesidad de desarrollar nuevas teorías específicas para la internacionalización de los servicios (Javalgi y Martín, 2007).

En este trabajo, basándonos en el paradigma OLI de Dunning (1977), trataremos de conocer cuáles son algunas de las ventajas de propiedad y de localización para las empresas turísticas situadas en Andalucía, sin hacer alusión a las ventajas de internalización. Algunas de las ventajas de propiedad se muestran en el cuadro que aparece a continuación y algunas de las ventajas de localización se especifican en el siguiente.

Tabla 2

Ventajas de propiedad

\begin{tabular}{|l|l|}
\hline Tamaño \\
\hline Activos inmateriales \\
- Tecnología \\
- Innovación \\
- Marca \\
- Capital humano \\
- Imagen \\
\hline Financiación \\
\hline Competitividad \\
- Precios \\
- Productividad \\
- Costes de producción \\
- Calidad
\end{tabular}

Fuente: Elaboración propia.

Por otra parte, respecto a las formas de suministrar el servicio, El GATS (General Agreement on Trade in Services), teniendo en cuenta el criterio de movilidad del productor y/o consumidor, considera las siguientes formas de entrada en países extranjeros:

Modo 1. Transacciones internacionales sin movimientos de proveedores ni clientes. Son esencialmente realizadas a través de redes de telecomunicación. La información y consulta de bancos de datos, así como las actividades financieras se encuentran dentro de esta categoría.

Modo 2. Transacciones internacionales con movimiento de clientes hacia el país del proveedor para obtener allí el servicio. Éstas conciernen especialmente al turismo, servicios sanitarios, educación, etc.

Modo 3. Transacciones internacionales en las que el proveedor abre una sucursal en el país del cliente para suministrar allí sus servicios. Todos los servicios pueden estar a priori relacionados con este tipo de intercambio internacional. Pueden ser incluidos hoteles, restaurantes, servicios personales, servicios a empresas, comercio, etc.

Modo 4. Transacciones internacionales con movimientos de proveedores hacia el país del cliente para hacer entrega del servicio. Son, principalmente, los casos de consultoría, servicios legales y servicios profesionales, en general, así como el transporte.

En general, las empresas turísticas pueden adoptar el modo 2, en el que el cliente se traslada hacia el país oferente y obtiene allí su servicio. Tanto los servicios de alojamiento, de comida y bebida y agencias de viaje pueden elegir esta vía de internacionalización. Además, en el caso de estas últimas, las nuevas tecnologías de la información le han permitido ofertar el servicio desde el país en el que estén localizadas y que el cliente lo pueda contratar desde su país de origen. Este caso sería un modo de internacionalización en que en la contratación de servicio no sería necesario la misma localización del oferente y el demandante (modo 1).

Otra vía de internacionalización sería la apertura de establecimientos turísticos en el exterior (modo 3). Por las propias características de los servicios de alojamiento y de comida y bebida, en el que la provisión del servicio y el consumo se ha de realizar simultáneamente en el mismo lugar y en el mismo tiempo, o lo que es lo mismo, en el que deben coincidir oferente y demandante, la empresa tiene 
que acudir obligatoriamente a la inversión directa extranjera como forma de internacionalización u a otras formas como joint ventures, franquicias, etc.

Tabla 3

Ventajas de localización de la inversión directa extranjera

\section{Contexto cultural e histórico}

* Idioma

* Calidad de vida del país

* Cultura empresarial

* Relaciones históricas entre países

* Sensibilidad social del país receptor ante la aparición de inversores extranjeros

\section{Dotación de recursos (básicos y avanzados)}

* Disponibilidad de recursos físicos

- Materias primas

- Localización geográfica

- Tamaño del país

- Clima

- Costas

- Parajes naturales

* Disponibilidad de mano de obra

* Infraestructuras

* Dotación tecnológica

Características de los mercados

* Tamaño del mercado

* Crecimiento del mercado

* Acceso al mercado

* Estabilidad económica

* Estabilidad política

Sistema económico y político

\section{Política Económica}

* Barreras comerciales

* Tipos de cambio

* Integración en áreas económicas supranacionales

* Política fiscal

* Mercado de trabajo:

- Costes laborales

- Cualificación de los trabajadores

- Productividad

Fuente: Muñoz Guarasa, M. (1999: 278)

\section{METODOLOGÍA}

La metodología empleada para conocer algunos de las ventajas de propiedad y de localización de las empresas turísticas del Sur de España es una encuesta realizada a una muestra de 130 compañías de internacionalizadas. Esta encuesta es una parte de una más amplia donde se han estudiado también las empresas de servicios intensivos en conocimiento (-Knowledge intensive services- KIS) (256 empresas) como contraposición de las anteriores por el tipo de servicios más avanzados que representan estas últimas.

Población objeto de estudio: El universo muestral está formado por empresas andaluzas englobadas dentro de las siguientes ramas de actividad de acuerdo con la Clasificación Nacional de Actividades Económicas (CNAE) 2009: Alojamiento (55), Servicios de comida y bebida (56) y Agencias de viajes (79). 
Ámbitos de la encuesta:

- Ámbito poblacional: Empresas internacionalizadas correspondientes a las ramas de actividad anteriormente señaladas.

- Ámbito territorial o geográfico: Empresas internacionalizadas que ejercen su actividad productiva en el territorio andaluz.

- Ámbito temporal: La encuesta se ha llevado a cabo en los meses de abril, mayo, junio y julio de 2011.

Unidades de encuesta: El conjunto de unidades que forman la muestra son empresas internacionalizadas andaluzas de las ramas de interés anteriormente definidas. Para la identificación de empresas que cumplieran los requisitos de la muestra y su selección se han utilizado diversas bases de datos como ICEX (Instituto Español de Comercio Exterior), Extenda (Agencia Andaluza de Promoción Exterior), base de datos de las Cámaras de Comercio, E-Informa y Axesor.

Método de muestreo: Se realiza un muestreo estratificado con afijación proporcional, tomando dos estratos: uno para empresas intensivas en conocimiento y otro para los servicios turísticos (códigos 55, 56 y 79$)$.

Selección de la muestra: La selección de la muestra de empresas internacionalizadas se realiza a partir de un proceso de estratificación, por el que se determina el tamaño muestral final. La estratificación divide la población en subgrupos o estratos cuyos elementos son homogéneos entre sí con el objeto de llevar a cabo selecciones separadas en cada uno de ellos. Su finalidad es controlar la representatividad de la muestra y determinar, -en función del estrato-, el número de unidades muestrales en cada uno de ellos.

Tamaño de la muestra y métodos de muestreo: Para la realización de un trabajo de campo sobre empresas internacionalizadas del sector servicios en Andalucía, es necesario extraer una muestra del universo de población de empresas. Según datos del Instituto Nacional de Estadística (INE) basados en el Directorio Central de Empresas (DIRCE), en el año 2010 existían en Andalucía un total de 173.434 empresas distribuidas del siguiente modo por ramas de actividad:

Tabla 4. Empresas por código CNAE09 en Andalucía en 2010

$\begin{array}{lr}55 \text { Servicios de alojamiento } & 2.956 \\ 56 \text { Servicios de comidas y bebidas } & 45.233 \\ 79 \text { Actividades de agencias de viajes, operadores turísticos, } & 2.005 \\ \text { operadores turísticos, servicios de reservas y actividades relacionadas con los mismos } & 123.240 \\ \text { Resto de empresas KIS internacionalizadas } & \text { Total } \mathbf{1 7 3 . 4 3 4}\end{array}$

Fuente: DIRCE. Elaboración propia

Las fuentes oficiales no publican datos acerca del número de empresas internacionalizadas en Andalucía. Para determinar el tamaño de muestra, hay que concretar el nivel de dispersión de las respuestas. Dado a que se desconoce el porcentaje exacto de empresas que se dedican a los mercados exteriores, suponemos la dispersión más desfavorable, en la que $\mathrm{P}$ y Q son igual a 0,5 , es decir, la varianza se hace máxima. Esto no implica un gran incremento en el tamaño muestral y es la opción más utilizada en la práctica. Se estima un tamaño de muestra de 386, con un nivel de confianza del 95 por 100 y un error máximo admisible de 0,05 , distribuida mediante afijación proporcional. El muestreo empleado es un muestreo aleatorio estratificado, donde el tamaño de los estratos es: 
Tabla 5. Estratos de la muestra

\begin{tabular}{lc}
\hline Estrato & $\mathbf{N}$ \\
Empresas turísticas & $\mathbf{1 3 0}$ \\
Resto de empresas internacionalizadas & 256 \\
Total & $\mathbf{3 8 6}$ \\
\hline $\begin{array}{l}\text { Los tamaños muestrales de los estratos oscilan entre el 90\% y 95\% de confianza. Errores } \\
\text { máximo admisibles inferiores al 8\% }\end{array}$ \\
\hline Fuente: Elaboración propia
\end{tabular}

\section{Descripción de la encuesta}

El cuestionario está dividido en ocho bloques de información: Bloque 1: Información general sobre la empresa; Bloque 2: Mercados internacionales; Bloque 3: Ventajas de propiedad; Bloque 4: Ventajas de localización; Bloque 5: Barreras; Bloque 6: Ayudas; Bloque 7: Nuevas tecnologías; Bloque 8: Innovación. El objetivo principal de análisis de nuestro estudio son los factores determinantes en el proceso de internacionalización que está centrado en el bloque 3 y bloque 4 de la encuesta y los aspectos generales del proceso de internacionalización en los bloques 1 y 2.

Trabajo de campo

La recogida de información se ha realizado mediante la concertación previa de citas con los empresarios, personal de gerencia o responsables de los departamentos de internacionalización. Se ha contado con un equipo de encuestadores distribuido por el territorio andaluz. Éstos se han encargado de cerrar citas con los responsables de las empresas objetivo para una posterior visita en el que se presentaba el proyecto y se procedía a la realización de la encuesta. La distribución de los encuestadores ha sido la siguiente: Dos encuestadores en: Almería, Córdoba, Granada y Jaén; Un encuestador en Málaga y un encuestador en: Cádiz, Huelva y Sevilla.

\section{RESULTADOS}

\subsection{Empresas de servicios turísticos internacionalizadas por ramas de actividad}

El sector turístico andaluz oferente de servicios a clientes extranjeros presenta en conjunto una estructura sectorial poco diversificada $\mathrm{y}$, por lo tanto, muy dependiente de ciertas actividades desarrolladas por esta industria. Las empresas que conforman el sector producen fundamentalmente servicios de alojamiento (hoteles, albergues, pensiones, etc.) y representan un 53,1 por 100 del total. En un segundo nivel, con una cuota de participación del 30,8 por 100, se sitúan las que centran su actividad en el subsector de la manutención, comida y bebida. Sin embargo, son minoría aquellas menos intensivas en capital físico, pero fundamentales para una adecuada comercialización del producto turístico andaluz, las agencias de viajes, que sólo representan un 16,1 por 100 del total de entidades encuestadas.

\section{Figura 1}

Distribución de las empresas internacionalizadas del sector turístico andaluz

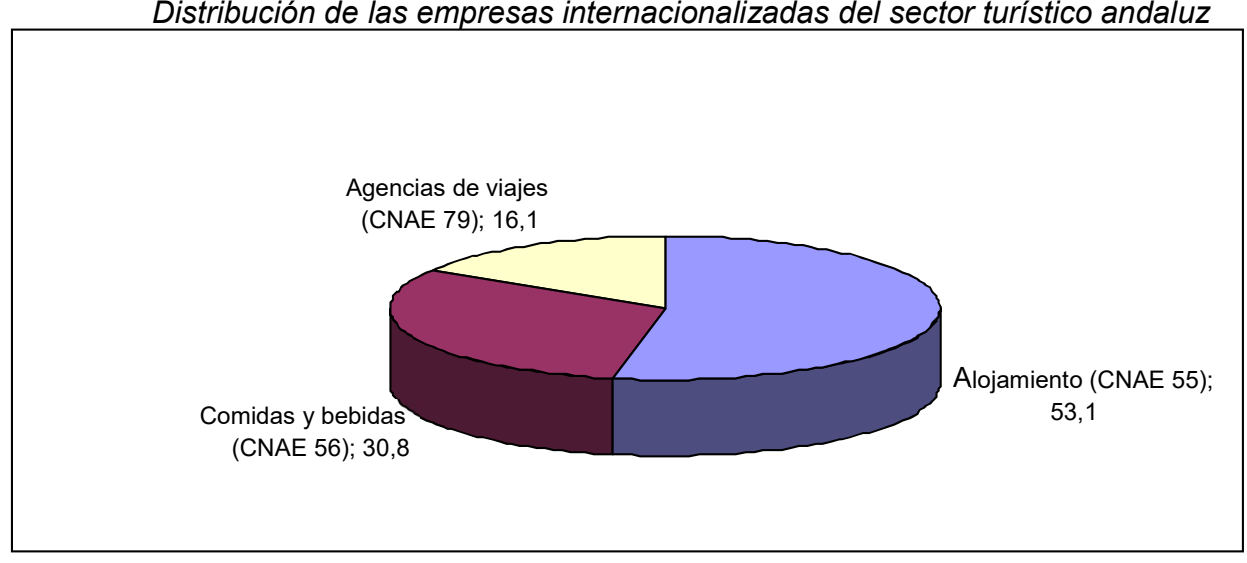

Fuente: Elaboración propia 


\subsection{Características de las empresas}

Identificada la composición del sector turístico andaluz internacionalizado, en este apartado se profundiza en el análisis de las características de estas empresas y en conocer los recursos de los que disponen para competir.

La dimensión de las unidades productivas es un factor especialmente relevante por cuanto condiciona sus posibilidades de actuación en mercados nacionales y transnacionales. El tamaño de las empresas turísticas se vincula fundamentalmente con el poder de negociación de los proveedores de servicios en relación con los grandes tour-operadores internacionales (Camisón, 1996). La empresa turística andaluza presenta, en conjunto, una dimensión reducida, en concordancia con lo que ocurre a nivel nacional, de acuerdo con los resultados obtenidos por Valls $(2009$, p. 163) que muestran que la causa de que muchos grupos españoles no se hayan abierto al exterior es su tamaño reducido. Concretamente el 60 por 100 de las entidades turísticas andaluzas encuestadas se clasifican como microempresas al tener menos de 10 trabajadores, siendo un 33,1 por ciento las de pequeña dimensión y un 6,9 por 100 medianas. Cuando se analiza la facturación, se comprueba que más del 75 por 100 ingresan menos de 2 millones de euros, lo que corrobora la escasa dimensión.

\begin{tabular}{lcc}
\hline \multicolumn{3}{c}{ Tabla 6. Número de empleados que tiene la empresa } \\
\hline Entre 1-9 trabajadores & Frecuencia & Porcentaje (\%) \\
Entre 10-49 trabajadores & 78 & 60,0 \\
Entre 50-250 trabajadores & 43 & 33,1 \\
Más de 250 & 9 & 6,9 \\
NC & 0 & - \\
Total & 0 & - \\
\hline Fuente: Elaboración propia & $\mathbf{1 3 0}$ & $\mathbf{1 0 0 , 0}$
\end{tabular}

A partir de los datos expuestos y de acuerdo con los argumentos referidos, es posible intuir que el sector cuenta con dificultades de carácter estructural que limitan su capacidad para operar y defender su producto en el exterior. En opinión de Rastrollo (2000: 136) las pequeñas y medianas empresas turísticas y, en general, el turismo independiente, no tiene una dimensión óptima para el establecimiento de redes comerciales que les permitan explotar los mercados de forma eficaz y eficiente y, de hecho, la comercialización de productos turísticos andaluces en el exterior se realiza mayoritariamente por grandes compañías que operan a nivel mundial.

Las empresas turísticas de la región tienen a los extranjeros como principales clientes. Más del 60 por 100 de las compañías que conforman el sector contratan regularmente con demandantes que residen fuera de las fronteras nacionales (tabla 7). Una situación que se corresponde, además, con el hecho de que exista en la región un alto porcentaje de compañías que llevan atendiendo el consumo de clientes extranjeros desde hace más de 20 años y que sean un 56 por 100 las que exporten sus servicios desde 2000 (tabla 12).

\begin{tabular}{lcc}
\hline & \multicolumn{3}{c}{ Tabla 7. ¿Regularmente realiza actividades de exportación de sus servicios? } \\
\hline & Frecuencia & Porcentaje (\%) \\
Sí & 80 & 61,5 \\
No & 50 & 38,5 \\
Total & $\mathbf{1 3 0}$ & $\mathbf{1 0 0 , 0}$ \\
\hline
\end{tabular}

Fuente: Elaboración propia

Los establecimientos turísticos andaluces, mayoritariamente de escasa dimensión, con una facturación de menos de 2 millones de euros, en más de un 75 por 100 de los casos analizados (tabla 8), responden fundamentalmente a las necesidades de consumidores finales procedentes del extranjero, pues sólo un 10,8 por 100 de su producción final resulta ser inputs intermedios para otras empresas (tabla 9). 


\begin{tabular}{|c|c|c|}
\hline & Frecuencia & Porcentaje $(\%)$ \\
\hline Menos de 2 millones de euros & 100 & 76,9 \\
\hline Entre 2 y 10 millones de euros & 6 & 4,6 \\
\hline Entre 10 y 50 millones de euros & 3 & 2,3 \\
\hline Más de 50 millones de euros & 0 & - \\
\hline $\mathrm{NC}$ & 21 & 16,2 \\
\hline Total & 130 & 100,0 \\
\hline \multicolumn{3}{|l|}{ Fuente: Elaboración propia } \\
\hline \multicolumn{3}{|c|}{ Tabla 9. Sector de destino de sus ventas en el exterior } \\
\hline & Frecuencia & Porcentaje (\%) \\
\hline Consumidores finales & 94 & 72,3 \\
\hline Otras empresas & 14 & 10,8 \\
\hline $\mathrm{NC}$ & 22 & 16,9 \\
\hline Total & 130 & 100,0 \\
\hline
\end{tabular}

Frente a los resultados expuestos, son minoría las entidades turísticas que incorporan servicios importados en sus procesos de producción, sólo un 6,9 por 100 (tabla 10). Se trata principalmente de compras de paquetes turísticos ofertados por las compañías extranjeras, fundamentalmente procedentes de los países de la UE-15, los nuevos socios de la UE, economías del Norte de África y países desarrollados como Estados Unidos, Canadá, Japón y Australia.

\begin{tabular}{lcc}
\hline \multicolumn{3}{c}{ Tabla 10. Importación de servicios de otros países } \\
\hline Sí & Frecuencia & Porcentaje (\%) \\
No & 9 & 6,9 \\
Total & 121 & 93,1 \\
\hline
\end{tabular}

Fuente: Elaboración propia

Respecto a la antigüedad de la empresa existe un peso importante de empresas turísticas que llevan más de 20 años en funcionamiento (34,6 por 100). El resto de valores oscilan entre el 8,5 por 100 y el 16,2 por 100 (tabla 11 ).

\begin{tabular}{lcc}
\hline & Tabla 11. Antigüedad de las empresas & \\
\hline & Frecuencia & Porcentaje (\%) \\
Menos de 2 años & 13 & 10,0 \\
3 a 5 años & 15 & 11,5 \\
6 a 10 años & 21 & 16,2 \\
11 a 15 años & 19 & 14,6 \\
16 a 20 años & 11 & 8,5 \\
Más de 20 años & 45 & 34,6 \\
NC & 6 & 4,6 \\
Total & $\mathbf{1 3 0}$ & $\mathbf{1 0 0 , 0}$ \\
\hline Fuente: Elaboración propia & &
\end{tabular}


Con relación al tiempo que la empresa lleva ofertando sus servicios a no residentes (tabla 12) destacan las que llevan más de 20 años de antigüedad (33,8 por 100). Les siguen las empresas entre 610 años (16,3 por 100) y, en tercer lugar, se sitúan las más jóvenes, con antigüedad igual o menor a dos años (13,8 por 100). Esto significa que estas compañías tienen cierta antigüedad, lo que le puede conferir experiencia y Know-How y lo que les permite afrontar con ventajas el proceso de internacionalización.

\begin{tabular}{lcc}
\hline & Tabla 12. Antigüedad realizando actividades de exportación \\
\hline & Frecuencia & Porcentaje (\%) \\
Menos (igual) de 2 años & 11 & 13,8 \\
Entre 3 -5 años & 10 & 12,5 \\
$6-10$ & 13 & 16,3 \\
$11-15$ & 10 & 12,5 \\
$16-20$ & 8 & 10 \\
Más de 20 & 27 & 33,8 \\
NC & 1 & 1,3 \\
Total & 80 & 100,0 \\
\hline Fuente: Elaboración propia & &
\end{tabular}

Fuente: Elaboración propia

\subsection{Elección del modo de internacionalización y países de destino}

A tenor de la caracterización del sector turístico andaluz internacionalizado y de los grandes rasgos que informan sobre el posicionamiento competitivo de sus empresas, en este apartado se profundiza en las distintas formas de internacionalización del sector y en los principales mercados de destino en los que opera, de acuerdo con la información estadística que presentan las tablas 13 y 14 .

Hoy en día, las empresas andaluzas que ofertan sus servicios en el extranjero desarrollan su actividad internacional fundamentalmente a través de la exportación, siendo muy reducido el porcentaje de entidades que estiman de gran importancia el contar con un socio comercial en el extranjero (un 5,4 por 100) o que consideran relevante disponer de sucursales en el exterior (un 5,4 por 100). Preferentemente, las empresas turísticas andaluzas concentran su negocio exterior en operaciones contratadas por clientes extranjeros en su país de origen, que posteriormente se desplazan hasta Andalucía para satisfacer su demanda. No obstante, la venta directa del producto ofertado por la región también es considerada como de relevancia alta o muy alta por más del 34,6 por 100 de las empresas encuestadas (tabla 13). Por otra parte, las empresas turísticas andaluzas encuestadas no destacan otras formas más avanzadas de internacionalización como joint ventures o franquicias, otorgándoles un protagonismo nulo en un 99,2 por 100 y 98,5 por 100 de los casos, respectivamente (tabla 14), en consonancia con el estudio de Berbel Pineda (2007: 30).

Independientemente del canal de internacionalización empleado, el sector presenta una fuerte concentración espacial de su clientela internacional. Más de cuatro quintas partes de los turistas extranjeros que demandan productos andaluces proceden de la UE-15. En conjunto, se trata de mercados de fuerte competencia en los que reside un consumidor de elevada renta per cápita e importantes niveles de consumo, pero muy vulnerable a la situación de crisis económica que han vivido gran parte de las economías desarrolladas del mundo. La fuerte dependencia del sector turístico andaluz de los clientes con origen en las economías del norte de Europa, determina que la realidad económica de estos países condicione la propia dinámica del sector regional y, por lo tanto, sus cifras de negocio anuales. 


\begin{tabular}{|c|c|c|c|c|c|c|c|}
\hline & Nula & Baja & Media & Alta & $\begin{array}{l}\text { Muy } \\
\text { alta }\end{array}$ & & \\
\hline \multirow{5}{*}{$\begin{array}{l}\text { 1.Venta directa desde } \\
\text { Andalucía a los clientes } \\
\text { en otros países }\end{array}$} & \multirow[t]{5}{*}{53,8} & \multirow[t]{5}{*}{4,6} & \multirow[t]{5}{*}{6,9} & \multirow[t]{5}{*}{10,8} & \multirow[t]{5}{*}{23,8} & UE-15 & 85,5 \\
\hline & & & & & & $\begin{array}{l}\text { Países } \\
\text { ampliación }\end{array}$ & 6,7 \\
\hline & & & & & & Resto Europa & 1,7 \\
\hline & & & & & & Latinoamérica & 3,3 \\
\hline & & & & & & Asia & 3,3 \\
\hline \multirow{5}{*}{$\begin{array}{l}\text { 2.Los clientes extranjeros } \\
\text { vienen a Andalucía }\end{array}$} & \multirow[t]{5}{*}{2,3} & \multirow[t]{5}{*}{3,8} & \multirow[t]{5}{*}{5,4} & \multirow[t]{5}{*}{35,4} & \multirow[t]{5}{*}{52,3} & UE-15 & 88,1 \\
\hline & & & & & & $\begin{array}{l}\text { Ampliación } \\
\text { UE-27 }\end{array}$ & 4,8 \\
\hline & & & & & & $\begin{array}{l}\text { Resto de } \\
\text { Europa }\end{array}$ & 2,4 \\
\hline & & & & & & $\begin{array}{l}\text { Otros países } \\
\text { des. }\end{array}$ & 1,6 \\
\hline & & & & & & Asia & 2,4 \\
\hline \multirow{4}{*}{$\begin{array}{l}\text { 3. Sucursal en el exterior } \\
\text { donde ofrece sus } \\
\text { servicios }\end{array}$} & \multirow[t]{4}{*}{90,0} & \multirow[t]{4}{*}{1,5} & \multirow[t]{4}{*}{0,8} & \multirow[t]{4}{*}{2,3} & \multirow[t]{4}{*}{5,4} & UE-15 & 69,2 \\
\hline & & & & & & Mercados Prox & 7,7 \\
\hline & & & & & & Latinoamérica & 15,4 \\
\hline & & & & & & Asia & 7,7 \\
\hline \multirow{4}{*}{$\begin{array}{l}\text { 4.Traslado de algún/os de } \\
\text { los miembros de su } \\
\text { empresa a otro país/es } \\
\text { para ofrecer su servicios }\end{array}$} & \multirow[t]{4}{*}{89,2} & \multirow[t]{4}{*}{0,5} & \multirow[t]{4}{*}{2,3} & \multirow[t]{4}{*}{2,3} & \multirow[t]{4}{*}{5,4} & UE-15 & 78,6 \\
\hline & & & & & & $\begin{array}{l}\text { Otros países } \\
\text { des. }\end{array}$ & 7,1 \\
\hline & & & & & & Latinoamérica & 7,1 \\
\hline & & & & & & Asia & 8,3 \\
\hline
\end{tabular}

Fuente: Elaboración propia 
Tabla 14. Importancia de los diferentes canales de ventas internacionales (\%)

\begin{tabular}{|c|c|c|c|c|c|c|c|}
\hline & Nula & Baja & Media & Alta & Muy alta & & \\
\hline \multirow[t]{4}{*}{ Exportaciones } & \multirow[t]{4}{*}{60,8} & \multirow[t]{4}{*}{1,5} & \multirow[t]{4}{*}{2,3} & \multirow[t]{4}{*}{11,5} & \multirow[t]{4}{*}{23,8} & UE-15 & 78,4 \\
\hline & & & & & & $\begin{array}{l}\text { Ampliación } \\
\text { UE-27 }\end{array}$ & 9,8 \\
\hline & & & & & & $\begin{array}{l}\text { Resto } \\
\text { Europa }\end{array}$ & 3,9 \\
\hline & & & & & & Asia & 7,8 \\
\hline Licencias & 94,6 & - & - & - & 5,4 & UE-15 & 100,0 \\
\hline \multirow[t]{2}{*}{ Sucursales en el exterior } & \multirow[t]{2}{*}{92,3} & \multirow[t]{2}{*}{1,5} & \multirow[t]{2}{*}{0,8} & \multirow[t]{2}{*}{1,5} & \multirow[t]{2}{*}{3,8} & UE-15 & 90,0 \\
\hline & & & & & & Asia & 10,0 \\
\hline \multirow{2}{*}{$\begin{array}{l}\text { Participación en una } \\
\text { empresa extranjera }\end{array}$} & \multirow[t]{2}{*}{90,8} & \multirow[t]{2}{*}{0,8} & \multirow[t]{2}{*}{3,1} & \multirow[t]{2}{*}{ - } & \multirow[t]{2}{*}{5,4} & UE-15 & 91,7 \\
\hline & & & & & & Asia & 8,3 \\
\hline Joint ventures & 99,2 & - & - & - & 0,8 & UE-15 & 100,0 \\
\hline \multirow[t]{2}{*}{ Licitaciones } & \multirow[t]{2}{*}{98,5} & \multirow[t]{2}{*}{0,8} & \multirow[t]{2}{*}{ - } & \multirow[t]{2}{*}{ - } & \multirow[t]{2}{*}{0,8} & UE-15 & 50,0 \\
\hline & & & & & & Asia & 50,0 \\
\hline Franquicias & 98,5 & 1,5 & - & - & - & UE-15 & 100,0 \\
\hline Otras & 43,1 & 2,3 & 5,4 & 12,3 & 10,0 & UE-15 & 100,0 \\
\hline
\end{tabular}

Figura 2

Mercados de destino de las exportaciones del sector turístico andaluz en 2011

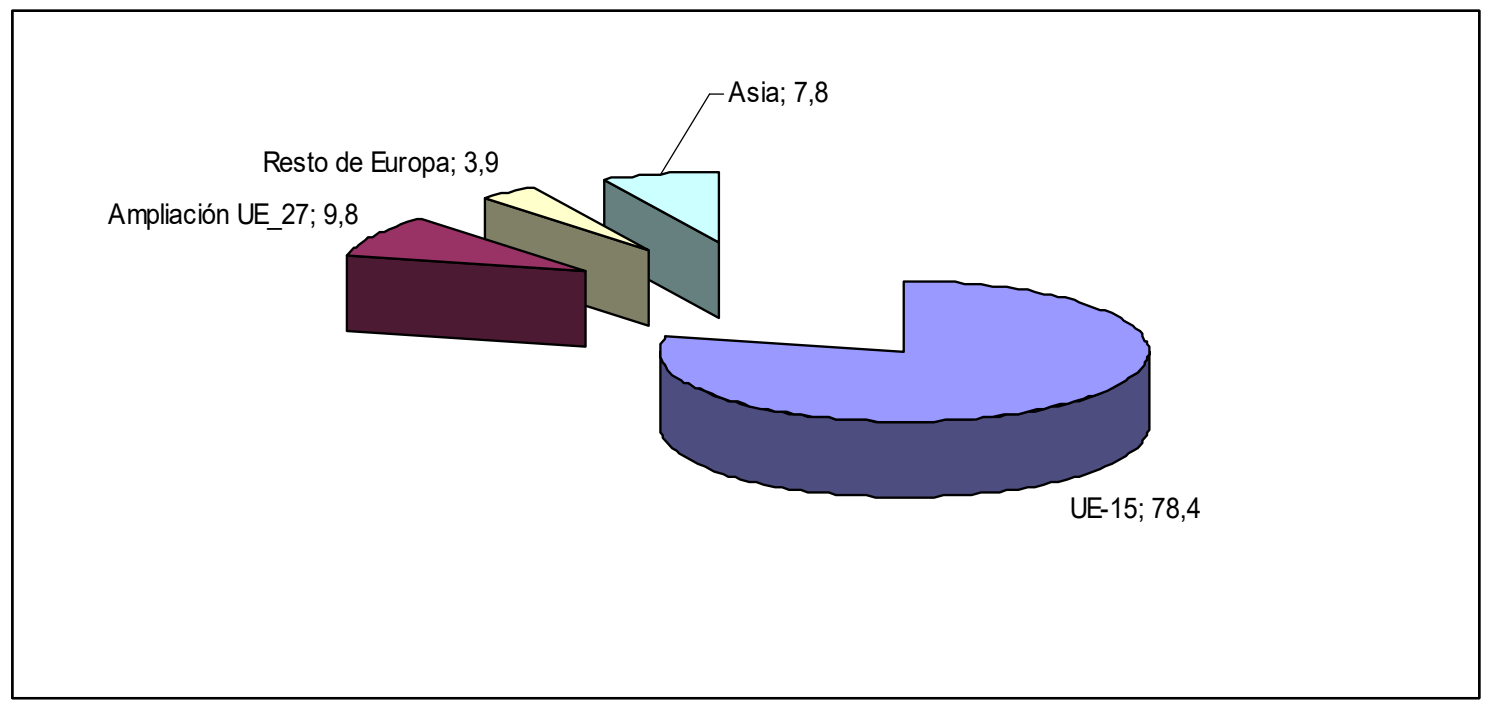

Fuente: Elaboración propia

\subsection{Factores determinantes}

\subsubsection{Ventajas de propiedad}

Los resultados de la encuesta sobre las ventajas de propiedad o competitivas en los servicios turísticos se recogen en la tabla 15. Existe una gran división de opiniones y esto se refleja en que los niveles en frecuencia entre los ítems de importancia alta y nula se dividen. Este hecho trae consigo que no se puedan percibir claramente cuáles son las ventajas competitivas más importantes. A pesar de ello, podemos observar que dentro de los activos propios de la empresa destaca la formación profesional sobre el resto, ya que un 50,8 por 100 de las empresas le otorgan una relevancia alta o muy alta; la siguiente ventaja es la capacidad directiva y de gestión con un 43,8 por 100 que le conceden esa misma importancia. 
Respecto a los mayores niveles de competitividad sobresale la calidad de los servicios a los cuales el 71,5 por 100 de las empresas la consideran de gran relevancia. También la competitividad en precios destaca con un 54,6 por 100 con la misma valoración. Las ventajas relativas al posicionamiento del mercado como imagen y cuota de mercado son consideradas por un 63,1 por 100 de las empresas y un 46,2 por 100, respectivamente de importancia alta y muy alta. Ramón (2002a: 237) pone de manifiesto que la imagen de marca y la comercialización determinan el éxito del sector hotelero mundial. Sin embargo, no constituían ventajas competitivas para las empresas hoteleras españolas, al menos hasta el momento de realización de ese trabajo. Por el contrario, hay que señalar que algunas ventajas como la dimensión de la empresa o la mayor capacidad financiera son consideradas por las compañías turísticas andaluzas poco representativas para su proceso de internacionalización.

\begin{tabular}{|c|c|c|c|c|c|c|}
\hline \multicolumn{7}{|c|}{ Tabla 15. Ventajas de propiedad } \\
\hline & & Nula & Baja & Media & Alta & $\begin{array}{l}\text { Muy } \\
\text { Alta }\end{array}$ \\
\hline a) & Activos propios de la empresa & & & & & \\
\hline- & Tecnología & 29,2 & 18,5 & 29,2 & 7,7 & 15,4 \\
\hline- & Innovación & 27,7 & 12,3 & 25,4 & 15,4 & 19,2 \\
\hline- & Capacidad directiva y de gestión & 25,4 & 4,6 & 26,2 & 23,8 & 20,0 \\
\hline- & Formación profesional & 25,4 & 3,8 & 20,0 & 28,5 & 22,3 \\
\hline- & Dimensión de la empresa & 28,5 & 27,7 & 29,2 & 9,2 & 5,4 \\
\hline b) & Mayor capacidad financiera & 30,0 & 22,3 & 33,1 & 8,5 & 6,2 \\
\hline c) & Mayores niveles de competitividad & & & & & \\
\hline- & Productividad & 26,2 & 5,4 & 26,2 & 22,3 & 20,0 \\
\hline- & Competencia en precios & 23,8 & 5,4 & 16,2 & 23,1 & 31,5 \\
\hline- & Costes de producción & 25,4 & 6,2 & 33,1 & 18,5 & 16,9 \\
\hline- & Calidad de los servicios & 22,3 & 0,8 & 5,4 & 26,9 & 44,6 \\
\hline d) & Posicionamiento del mercado & & & & & \\
\hline- & Cuota de mercado & 23,1 & 8,5 & 22,3 & 20,8 & 25,4 \\
\hline- & Imagen & 21,5 & 3,1 & 12,3 & 27,7 & 35,4 \\
\hline e) & Otras & 56,9 & 0,8 & 3,1 & 2,3 & - \\
\hline
\end{tabular}

\subsubsection{Ventajas de localización}

No se aprecian con claridad las ventajas de localización que muestran las empresas como determinantes de su decisión de vender y/o producir sus servicios en otro país como consecuencia, quizá, del gran número de factores propuestos (véase tabla 16). Respecto a los factores relativos a las características del mercado otorgan una importancia alta y muy alta al tamaño del mercado el 50 por 100 de las empresas y al crecimiento del mercado un 62,5 por 100 y ninguna declara considerar una relevancia nula a ambos factores. Los factores de localización relativos al contexto cultural también son relevantes, tales como la calidad de vida del país y el idioma, de forma que en ambos casos el 75,0 por 100 los consideran de importancia alta y muy alta y, por su parte, a la cultura empresarial se la otorgan un 62,5 por 100 . Es de resaltar otros factores de localización como la proximidad a los clientes ( 75 por $100)$ y el seguimiento al cliente $(62,5$ por 100$)$. 


\begin{tabular}{|c|c|c|c|c|c|c|}
\hline & & Nula & Baja & Media & Alta & Muy Alta \\
\hline a) & Dotación de recursos & & & & & \\
\hline- & Bajo coste de la mano de obra & 37,5 & 25,0 & 25,0 & - & 12,5 \\
\hline- & Acceso al personal cualificado & 25,0 & 12,5 & 25,0 & 37,5 & - \\
\hline- & Infraestructuras & 12,5 & 25,0 & 25,0 & 37,5 & - \\
\hline- & Dotación tecnológica & 12,5 & 37,5 & 37,5 & 12,5 & - \\
\hline b) & Características del mercado & & & & & \\
\hline- & Tamaño del mercado & - & 25,0 & 25,0 & 12,5 & 37,5 \\
\hline- & Crecimiento del mercado & - & 37,5 & - & 37,5 & 25,0 \\
\hline- & Acceso al mercado interno & - & 25,0 & 37,5 & 25,0 & 12,5 \\
\hline- & $\begin{array}{l}\text { Forma de acceso a otros mercados } \\
\text { extranjeros }\end{array}$ & 12,5 & 25,0 & 25,0 & 25,0 & 12,5 \\
\hline
\end{tabular}

c) Factores institucionales

$\begin{array}{lllllll}\text { - } & \text { Favorable legislación del sector } & 25,0 & 37,5 & 25,0 & 12,5 & - \\ \text { - } & \text { Incentivos fiscales } & 37,5 & 25,0 & 25,0 & - & 12,5\end{array}$

d) Contexto cultural

$\begin{array}{lllllll}\text { - } & \text { Calidad de vida del país } & 12,5 & - & 12,5 & 62,5 & 12,5 \\ \text { - } & \text { Cultura empresarial } & 12,5 & - & 25,0 & 50,0 & 12,5 \\ \text { - } & \text { Idioma } & 12,5 & - & 12,5 & 62,5 & 12,5\end{array}$

e) Otras

\begin{tabular}{|c|c|c|c|c|c|c|}
\hline - & Ausencia de empresas locales fuertes & 25,0 & 25,0 & 12,5 & 12,5 & 25,0 \\
\hline - & Proximidad a los clientes & 12,5 & 12,5 & - & 37,5 & 37,5 \\
\hline- & Proximidad a los proveedores & 37,5 & 25,0 & 12,5 & 12,5 & 12,5 \\
\hline- & $\begin{array}{l}\text { Proximidad a la Universidad o centros } \\
\text { de investigación }\end{array}$ & 62,5 & 12,5 & 25,0 & - & - \\
\hline- & Proximidad a los cluster & 62,5 & - & 37,5 & - & - \\
\hline- & Seguimiento al cliente & 25,0 & - & 12,5 & 25,0 & 37,5 \\
\hline
\end{tabular}

Fuente: Elaboración propia

Ramón (2002a: 240) señala como factores de localización para el conjunto de España no sólo el atractivo del país de destino (playas, recursos naturales e históricos), sino también el papel del sector público del destino en tanto en cuanto que de su actuación depende el desarrollo de infraestructuras, las exigencias medioambientales, la estabilidad política, social y económico, la política hacia la inversión extranjera y la cualificación de los recursos humanos. Asimismo, la similitud cultural con España, la generación de economías de aglomeración que refuerzan las ventajas obtenidas por la internacionalización así como la posibilidad de diversificar mercados de origen se constituyen en factores relevantes para la localización hotelera vacacional. Por su parte, Furió y Alonso (2007: 41) asocian la inversión en el exterior de las empresas hoteleras a bajos niveles de riesgo-país, reducida o nula distancia cultural, mercados de elevado tamaño, economías con alto nivel de desarrollo y gran apertura exterior y alto potencial de crecimiento. 


\section{CONCLUSIONES}

La internacionalización de las empresas turísticas en Andalucía se está llevando a cabo de una forma pasiva, puesto que la principal vía han sido las exportaciones materializadas a través de la llegada de extranjeros a Andalucía que hacen uso de estos establecimientos. Sin embargo, quedan relegadas a un segundo plano otras formas de internacionalización más avanzadas como las sucursales en el exterior, joint-ventures, franquicias, etc. Este proceso de internacionalización viene limitado por algunos problemas estructurales que presentan estas empresas como la escasa dimensión, tanto en cuanto al número de trabajadores como a la facturación. Sin embargo, estas empresas se caracterizan por tener una cierta antigüedad, el 34, 6 por 100 nacieron hace más de 20 años y un 33,8 por 100, llevan exportando más de 20 años. Esto nos puede mostrar que las empresas que tienen más experiencia y, por tanto, mayor nivel de Know-How, son las que están inmersas en estos procesos de internacionalización.

Por otro lado, existe una gran concentración de clientes según su origen geográfico, de forma que más del 75 por 100 de ellos proceden de la UE-15. Si bien estos mercados se caracterizan por una elevada renta per cápita y grandes posibilidades de consumo, esta fuerte dependencia condiciona la evolución del sector turístico andaluz a la situación económica de los mismos.

Los factores determinantes del proceso de internacionalización que las empresas consideran son los siguientes: Las ventajas de propiedad más importantes que creen tener estas compañías son la calidad del servicio, seguida de la imagen y la competitividad en precios. También, pero en menor medida, destacan la cualificación del personal. Por su parte, las empresas que invierten en el exterior consideran como factores de localización, es decir, características del país de destino, en primer lugar, "la proximidad al cliente" y "el seguimiento al cliente". Igualmente, las variables relacionadas con las características de los mercados como el tamaño del mercado y de su crecimiento y también los relacionados con el contexto cultural e histórico del país como la calidad de vida del país, idioma y cultura empresarial son las más destacadas por estas empresas.

En definitiva, hay que poner de manifiesto que el proceso de internacionalización de las empresas turísticas situadas en Andalucía se encuentra en un estado incipiente y que durante los próximos años debería de estar más avanzado. Para ello sería necesario, en primer lugar, eliminar algunos problemas estructurales que presenta el sector como es la pequeña dimensión, ya que el mismo está liderado por pequeñas y medianas empresas. La redimensión del sector se podría alcanzar mediante fusiones y adquisiciones o con acuerdos de colaboración. Esto sería necesario, no sólo para ganar en competitividad internacional, sino también nacional. Por otra parte, para avanzar en el proceso de internacionalización habría que utilizar otras formas más activas y más comprometidas como la inversión en el exterior. Igualmente habría que diversificar hacia países menos desarrollados, por ejemplo, Norte de África, o Latinoamérica, con gran potencial de crecimiento de sus mercados. Así, el establecimiento en estos países permitiría a las empresas andaluzas explotar su Know-How a la vez que se beneficiarían de los factores de localización de dichos países.

Por último, hemos de poner de manifiesto que una extensión de esta investigación podría consistir en contratar las relaciones que se puedan producir entre las distintas ventajas de propiedad y localización con las características de las empresas.

\section{REFERENCIAS BIBLIOGRÁFICAS}

AGENCIA ANDALUZA DE PROMOCIÓN EXTERIOR (EXTENDA) (2011). Base de datos de empresas exportadoras. EXTENDA.

AXESOR (2011). Base de datos de empresas. AXESOR

BERBEL PINEDA, J.M. (2006). Influencia del modo de entrada en mercados exteriores sobre Export Performance: Aplicación a la Internacionalización de la Industria Hotelera. Tesis Doctoral- Sevilla: Universidad Pablo de Olavide. Sevilla.

BERBEL PINEDA, J.M. ; CRIADO GARCIA-LEGAZ, F. Y PUIG BLANCO, F. (2007). "Factores a considerar en la elección del modo de entrada para la internacionalización de la industria hotelera andaluza” en Revista de Estudios Empresariales. Segunda época, 1, pp. 5-37.

BHAGWATI, J. (1988). "Importance du commerce international des services pour le developpement economique" en L'Europe face a la nouvelle economie de services, pp. 17-48.

BODDEWYN, J.J.; HALBRICH, M.B. Y PERRY, A.C. (1986). "Service multinationals: conceptualization, measurement and theory" en Journal of International Business Studies, 17(3), pp. 41-57. 
BUCKLEY, P.; PASS, C.L. Y PRESCOTT, K. (1992). "The internationalization of services firms: a comparison with the manufacturing sector, Scandinavian International Business Review, 1, pp. 39-57.

CAMISÓN, C. (1996). "La empresa turística: un análisis estratégico", en A, Pedreño (dir.). Introducción a la economía del turismo, pp. 217-246. Madrid: Cívitas.

CÁMARAS DE COMERCIO DE ESPAÑA (2011). Base de datos de las Cámaras de Comercio. Cámaras de Comercio de España.

DUNNING, J.H. (1977). "Trade, location of economic activity and the MNE: A search for an eclectic approach" en B, Ohlin, P, Hesselborn, y P, Wiskman (eds.). The International Allocation of Economic Activity. London: Macmillam , pp. 395-419.

DUNNING, J.H. (1988). Explaining international production. London: Unwin Hyman.

DUNNING, J.H. (1993). Multinational enterprises and the global economy. Addison-Wesley.

EDVARDSSON, B.; EDVINSSON, L. Y NYSTROM, H. (1993). "Internationalisation in Service Companies" en The Service Industries Journal, 13(1), pp. 80-97.

eINFORMA (2011). Base de datos de empresas. eINFORMA.

ERRAMILLI, M.K. (1990). "Entry mode choice in Service Industries" en International Marketing Review, 7(5), pp. 50-62.

GUERRIERI, P. Y MELICIANI, V. (2007). "La producción e internacionalización de los servicios: Determinantes e implicaciones para la innovación y el crecimiento en Europa" en ICEX .Claves de la Economía Mundial, Madrid.

FURIO, E. Y ALONSO, M. (2008). "Las variables país e implantación exterior de la industria hotelera" en Boletín Económico de ICE, 2908, pp. 31-46.

HILL, T.P. (1977). "On goods and services" en Review of Income and Wealth, December.

INSTITUTO NACIONAL DE ESTADÍSTICA (2011). Directorio Central de Empresas (DIRCE) (2010). INE.

INSTITUTO NACIONAL DE ESTADÍSTICA (2012). Contabilidad Regional. INE.

JAVALGI, R. G. Y MARTíN, C.L. (2007): "Internationalization of services: identifying the building-blocks for future research" en Journal of Services Marketing, 21 (6), pp. 391-397.

JENÍCEK, V. (2007). "World trade with services in globalization processes" en Agric. Econ., 53, pp. 55-64.

JOHANSON, J. Y VAHLNE, J. (1990). The mechanism of Internationalisation" en International Marketing Review, 7(4), pp. 11-23.

KOLSTAD, I. Y VILLANGER, E. (2008). "Determinants of foreign direct investment in services" en European Journal of Political Economy, 24, pp. 518-533.

METCALFE, S. Y POTTS, J. (2007): "La internacionalización de los servicios: la perspectiva evolutiva" en Claves de la Economía Mundial, pp. 79- 87.

MINISTERIO DE ECONOMÍA Y COMPETITIVIDAD (2012A). Estadísticas de inversiones exteriores, Datalnvex.

Secretaría de Estado de Comercio. http://www.comercio.gob.es/es-ES/inversiones-

exteriores/estadisticas/Paginas/estadisticas-inversiones-exteriores-datainvex.aspx.

MINISTERIO DE ECONOMÍA Y COMPETITIVIDAD (2012B). Estadísticas de Comercio Exterior español, DataComex. Secretaría de Estado de Comercio. http://datacomex.comercio.es/principal_comex_es.aspx.

MUÑOZ-GUARASA, M. (1999). La inversión directa extranjera en España: factores determinantes. Madrid: Civitas.

MUÑOZ- GUARASA, M. (2005). "La internacionalización de los servicios en los países desarrollados". Comercio Exterior, 55 (8), pp. 692-700.

MUÑOZ-GUARASA, M. (2007). “La deslocalización de los servicios: ¿Mito o realidad?” en Revista de Economía Mundial, 16, pp. 57-78.

O`FARREL, P.N. Y WOOD, P.A (1996). "Internationalization of business services: an interregional analysis" en Regional Studies, 30, pp. 10-118

RAMASAMY, B. Y YEUNG, M. (2010). "The Determinants of Foreign Direct Investment in Services" en The World Economy, pp. 573-596.

RAMÓN, A. B. (2002a). La expansión internacional del sector hotelero español. Caja de Ahorros del Mediterráneo.

RAMÓN, A.B. (2002b). "Un análisis de las dimensiones del paradigma OLI en la internacionalización del sector hotelero español" en Información Comercial Española, 803, pp. 141-151.

RASTROLLO, M. A. (2000). "La empresa turística en Andalucía” en Boletín Económico de Andalucía. 28-29, pp. 128-140.

ROBERTS, J. (1998). Multinational Business Service Firms. Aldeshot: Ashgate.

SAMPSON, G.P. Y SNAPE, R.H. (1985). "Identifiying the Isues in Trade in Services" en World Economy, 8 (2), pp. $171-181$.

SÁNCHEZ-PEINADO, E.; PLA-BARBER, J. Y HÉBERT, L. (2007): “Strategic Variables That Influence Entry Mode Choice in Service Firms”. Journal of International Marketing, 15 (1), pp. 67-91.

SAPIR, A. (1993a). "Sectorial dimension" en European Economy, 3.

SAPIR, A. (1993b). "The structure of services in Europe: A conceptual framework" en European Economy, 3, pp. 83-98.

VALLS, J.F. (2009). "La internacionalización del sector hotelero: El caso español" en P. Puig-Bastard (coord.) et al, La expansión de la multinacional española: estrategias y cambios organizativos, Primer informe anual del Observatorio de la Empresa Multinacional Española, pp. 150-165.

VANDERMERWE, S. Y CHADWICK, M. (1989): "The internationalisation of Services" en The Service Industries Journal, 9 (1) 1, pp. 79-93. 